\title{
A record from an active asteroid preserved in a carbonaceous chondrite
}

\section{Xin Yang ( $\nabla$ xinyoung@uchicago.edu )}

University of Chicago https://orcid.org/0000-0001-5936-486X

\section{Romy Hanna}

The University of Texas at Austin https://orcid.org/0000-0002-2102-8040

\section{Andrew Davis}

University of Chicago

\section{April Neander}

University of Chicago

\section{Philipp Heck}

Field Museum of Natural History https://orcid.org/0000-0002-6319-2594

\section{Article}

\section{Keywords:}

Posted Date: February 2nd, 2022

DOI: https://doi.org/10.21203/rs.3.rs-1247961/v1

License: (c) (1) This work is licensed under a Creative Commons Attribution 4.0 International License. Read Full License

Version of Record: A version of this preprint was published at Nature Astronomy on August 11th, 2022. See the published version at https://doi.org/10.1038/s41550-022-01746-4. 


\section{Abstract}

Multiple particle ejection and redeposition events on the surface of asteroid 101955 Bennu were observed frequently by NASA's OSIRIS-REx mission and advanced our understanding of active asteroids. This finding motivated us to search for relicts of such activity in a Bennu analog - the carbonaceous chondrite Aguas Zarcas (AZ). Here we discovered compact fragments that were strongly shocked, redistributed on the parent body, and deposited into an unshocked lithology. While breccias in meteorites are common, this type of lithology has been rarely observed. However, it is consistent with the observed particle redeposition process on Bennu. In addition, we observed a match in the size distribution of the compact fragments with that of the Bennu ejecta, indicating that the same process operated on the AZ parent body. Our findings suggest that such mixing may have been unnoticed in most meteorites and that asteroid activity may be common but overlooked.

\section{Introduction}

NASA's Origins, Spectral Interpretation, Resource Identification, and Security-Regolith Explorer mission (OSIRIS-REx) observed particle ejection events on asteroid 101955 Bennu that constitutes the first observation of an active asteroid by an orbiting spacecraft ${ }^{1}$. Particles in the millimeter- to centimeter-size range are ejected from the asteroid surface with most falling back ${ }^{2}$. This process redistributes mass and mixes rocks with different geological histories from different locations and contributes to regolith gardening and breccia formation. Different histories include varying degrees of aqueous alteration, and local deformation and fracturing through impacts ${ }^{3}$ that form meteorite breccias. A large fraction of meteorite types are brecciated, composed of rock fragments cemented together. For example, almost all $\mathrm{Cl}$ and $\mathrm{CM}$ chondrites, mesosiderites, aubrites, more than $80 \%$ of HED meteorites, $23 \%$ of $\mathrm{H}$ and $\mathrm{L}$ chondrites, and $79 \%$ of $L L$ chondrites are breccias ${ }^{4,5}$. These breccias, samples mostly from asteroid surfaces and subsurfaces, provide the most direct record of surficial processes on asteroids. We suspected that a record of particle ejection and redeposition processes like what were observed on Bennu is preserved in some meteorite breccias.

We selected Aguas Zarcas (hereafter AZ), a CM2 meteorite that is similar in composition to asteroid Bennu based on the spectral data ${ }^{6}$. AZ is a breccia containing several lithologies such as brecciated CM, $\mathrm{C} 1, \mathrm{C} 1 / 2$, and metal-rich ones, which are the result of collisions and aqueous alteration ${ }^{7}$. We used mechanical disintegration to characterize the rock, followed by X-ray microtomography $(\mu \mathrm{CT})$ and scanning electron microscopy (SEM) (see Methods), then compared our data with the observations of Bennu. To quantify the global mass transport on Bennu-like asteroids with varying radii, we conducted a Monte Carlo analysis. We used all the results to infer that AZ most likely preserved a record of the recently observed particle ejection and reaccretion processes on a low-level active asteroid similar to Bennu.

\section{Results}


We found a compact lithology by disaggregating $\sim 79 \mathrm{~g}$ of AZ fragments randomly selected from a large and freshly broken hand-sample $(1.894 \mathrm{~kg})$ into fine powder with the freeze-thaw method. More than 10 sub-cm-sized compact fragments (3.2 wt\%) with a dull, black, smooth luster similar to the appearance of melt rock survived the disintegration (see Methods). The compact lithology is much more resistant to the mechanical breakdown and has a higher density $\left(\sim 2.7 \mathrm{~g} \mathrm{~cm}^{-3}\right)$ and compactness compared to the regular host lithology $\left(\sim 2.4 \mathrm{~g} \mathrm{~cm}^{-3}\right)$. Because of the low abundance of the compact lithology, we assumed that the randomly selected rocks are not compact and belong to the regular AZ lithology. We observed prominently visible deformation and a preferred orientation of chondrules in the $\mu C T$ and SEM data of compact AZ and Leoville and no such effect in regular AZ and Murchison (Fig. 1). Murchison is one of the best-studies meteorites without prevalent deformation, and Leoville is known as one of the most deformed chondrites showing the alignment of flattened chondrules ${ }^{8,9}$. They were used as two endmembers to constrain the deformation of AZ.

To assess and quantify the type and strength of deformation, we outlined chondrules in the $\mu$ CT data set and fitted ellipsoids following an established method ${ }^{10}$ and used axial ratios, fabric parameters, and shape analysis with ternary diagrams ${ }^{11,12}$. Fabric is the geometric arrangement of components in a rock. In our case it refers to the spatial arrangement of chondrules and their preferred orientation that manifests itself as elongated (rod shape, lineation) or flattened (disc shape, foliation) chondrules. This is also referred to as foliation as a term for any type of planar fabric in a rock. Fabric parameters $(K$ and $C)$ are defined by a set of direction vectors of axes of the best-fit ellipsoids of chondrules and can be used to distinguish deformation type (lineation or foliation) and quantify the deformation intensity ${ }^{13} . K<1$ for the longest axes set and $K>1$ for the shortest axes set demonstrates a foliation, and $K>1$ for both axes sets indicates a lineation. For the strength parameter $C$, higher values indicate stronger fabrics.

The average axial ratios of fitted ellipsoids of chondrules increase from regular AZ and Murchison to compact $\mathrm{AZ}$ and Leoville, which is consistent with the result of $C$ parameters (Supplementary Table 1). The fabric strength varies from 'moderately weak' to 'moderately strong' with $C$ of longest axes ranging from 0.75 to 2.53 and $C$ of shortest axes ranging from 1.04 to 2.65. These parameters are highest for compact $A Z$ and Leoville and are lowest for regular AZ and Murchison, indicating that the former two are strongly shocked and that the latter are weakly shocked. $K$ parameters for the longest axes in compact $A Z$ and Leoville range from 0.09 to 0.40 while those for the shortest axes range from 1.97 to 13.06 , arguing that the fabrics in compact AZ and Leoville are both foliations. That is, the chondrules are more flattened than elongated, though the difference cannot be distinguished by 2D analysis (Fig. 1).

Meanwhile, in the ternary diagram (Fig. 2) that plots an object's shape as a function of a perfect sphere, elongated rod, and platy disc shape, we note two patterns of chondrule shape distribution. One group, represented by regular AZ and Murchison, has the majority of their chondrule shapes located in the top 'equant shape' sub-triangle. The other group, represented by compact $A Z$ and Leoville, has a significant number of points in the areas signifying more deformed shapes ( $44.0 \%$ for compact $A Z, 64.6 \%$ for Leoville, $17.4 \%$ for regular $A Z, 19.0 \%$ for Murchison). Combining these indices above, we infer that the deformation intensity sequence is regular $A Z<$ Murchison $<$ compact $A Z<$ Leoville. An exception of these 
samples is regular AZ fragment RF-3 that displays a higher axial ratio and $C$ parameter compared to other regular AZ fragments and is closer to compact AZ (Supplementary Fig. 1). Therefore, we classify RF-3 as a compact AZ fragment. The classification is not binary. RF-3 is more deformed than regular ones but may not be as deformed as the other compact fragments. This deformation sequence is also reflected by the fragments' average densities $\left(\sim 2.7 \mathrm{~g} \mathrm{~cm}^{-3}\right.$ for compact $\mathrm{AZ}, \sim 2.4 \mathrm{~g} \mathrm{~cm}^{-3}$ for regular $\mathrm{AZ}$, and $2.55 \mathrm{~g}$ $\mathrm{cm}^{-3}$ for RF-3).

Besides deformation, fractures and veins are often used to investigate meteorites' stress histories in shock events 3,14 . In the polished section of compact fragment CF-10, we observed six major fractures (0.5-6 mm long, 7-20 $\mu \mathrm{m}$ wide) and many minor fractures (several hundred $\mu \mathrm{m}$ long, $\sim 2 \mu \mathrm{m}$ wide) in the matrix, and some small fractures within chondrules, some of which are filled with metal sulfide veins oriented independent of the chondrule flattening direction (10-20 $\mu \mathrm{m}$, Fig. 1). In contrast, the fractures in the matrix are all empty and mostly parallel or subparallel to the direction of the chondrule elongation. We also examined the $\mu C T$ data of regular $A Z$ and found several unfilled fractures $(10-20 \mu \mathrm{m}$ wide, 1-2 mm long) without any preferred orientation (Supplementary Fig. 2).

Fractures and deformation are common in chondrites ${ }^{15}$, and an impact origin is supported by an increasing amount of evidence such as the correlation between shock stages and aspect ratios of chondrules, noncoaxial strain, and the abundance of unfilled vs. fractures filled with secondary minerals $8,10,14,16$. Meanwhile, fracturing in the compact AZ matrix is approximately in the same orientation as chondrule flattening. Therefore, we propose that the same generation of impact events caused the shock effects observed in the compact AZ lithology and that no significant aqueous alteration occurred after the shock, otherwise, these fractures would be filled with secondary precipitates. Metal sulfide veins are not common in CM chondrites but are often seen in ordinary and some CV meteorites ${ }^{16,17}$ that are likely to have experienced collisions and heating events. For sulfide veins in chondrules in compact $\mathrm{AZ}$, one possible scenario is that an impact opened fractures in chondrules and preferentially melted sulfide filled fractures to form dike-like veins. While such veins from impacts are usually large and cross into the matrix, the veins in compact $A Z$ are thin (10 to $20 \mu \mathrm{m}$ wide) and only exist in chondrules. Thus, a single collision is less likely to have formed the veins observed here. In another, more plausible scenario, fracturing occurred first in metal-rich chondrules in the solar nebula before accretion or during accretion to the parent body, and sulfide veins were formed simultaneously by shock mobilization or later by thermal or aqueous alteration. The supportive evidence for this is that these veins are not well orientated with the cracks in the matrix. And based on cross-cutting relationships and because fractures outside of chondrules are unfilled, we infer that the chondrule veins formed prior to the impact-induced matrix fractures. Otherwise, we would expect to see all fractures, including those in the matrix, to be filled.

We can exclude deformation by lithostatic pressure through burial in the regolith: we modeled the lithostatic pressure for chondritic bodies with varying sizes (Supplementary Fig. 3) and find that the maximum pressure in the center of a Ceres-like asteroid is only about $0.5 \mathrm{GPa}$. This is a much deeper 
depth than a plausible burial depth and a much lower pressure than needed to explain the deformation of compact AZ (see the section below, shock pressure estimate).

Traditionally, shock effects in olivine are used to determine the shock pressure, but they may not reflect the shock history of CMs well because the abundant matrix ( 70 vol\%) in CM meteorites can significantly attenuate a shock wave to a low intensity such that it cannot affect olivine crystals ${ }^{3}$. Suitable indicators of shock pressure in $\mathrm{CMs}$ include chondrule flattening and fractures. First, empirical relationships between chondrule aspect ratio and shock pressure were established in impact shock events ${ }^{8,18}$. Based on these relationships for $\mathrm{CV}$ and $\mathrm{CM}$ chondrites (Fig. 3), we determined shock pressures for Leoville and compact AZ as $\sim 17 \mathrm{GPa}$ and $\sim 19 \mathrm{GPa}$, respectively. The published shock stage for Leoville is S3, corresponding to a shock pressure of $15-20 \mathrm{GPa}$ in a single impact ${ }^{16,19}$. The consistency between the shock pressure determined from chondrule aspect ratio and published shock stage for Leoville demonstrates the suitability of this method. Second, the existence and density of fractures are qualitative indicators of shock pressure. In shock events of Murchison ${ }^{8}$, the recovered sample showed that the fracture $(<5 \mu \mathrm{m})$ density in the matrix increased slightly when the pressure increased up to $10 \mathrm{GPa}$ and that fractures became wider $(20 \mu \mathrm{m})$ and more preferentially orientated at $21 \mathrm{GPa}$, then olivine grains showed undulatory extinction and planar fractures that were consistent with shock stage S3. The occurrence of 2-20 $\mu \mathrm{m}$-wide and unfilled fractures in compact AZ matrix (some pass through the flattened chondrules) parallel or at a low angle to the direction of chondrule deformation is also consistent with a shock pressure of 15-20 GPa.

Bischoff et al. ${ }^{4}$ explored two types of impact collisions. Accretionary impacts happened during the accretion of asteroids at relatively slow speeds (typically less than a few hundred $\mathrm{m} \mathrm{s}^{-1}$ ). Hypervelocity impacts occurred after asteroidal orbits were dynamically excited when asteroids collided at speeds of a few $\mathrm{km} \mathrm{s}^{-1}$. Tomeoka et al. ${ }^{8}$ display a pressure-particle velocity diagram showing that an impactor with a speed $<1 \mathrm{~km} \mathrm{~s}^{-1}$ cannot generate pressure greater than $10 \mathrm{GPa}$. Meanwhile, Lindgren et al. ${ }^{14}$ concluded that most of the meteorites that contain high aspect ratio chondrules provide independent evidence of hypervelocity impact in the form of shock fractures that we also observed in compact AZ fragments. Therefore, we infer that the compact lithology must have experienced at least one hypervelocity impact.

\section{Discussion}

AZ is highly brecciated with multiple lithologies that are thought to be the result of different degrees of aqueous alteration and impact modification ${ }^{7}$. Nevertheless, the two distinct types of AZ in a small volume cannot be explained as products of different degrees of aqueous alteration or heterogeneous shocking effect within such close $(\mathrm{mm})$ proximity, but rather suggest necessary transport of the compact fragments into an undeformed lithology before final lithification. On most asteroids, meteoroid impacts are commonly responsible for the shock. Shock events cause identical petrofabrics among all lithologies in a rock ${ }^{15}$, even in different hand specimens, akin to Leoville. Variably shocked materials within one sample are sometimes observed in impact melt rocks/breccias containing shock-melted clastic material 
and unmelted but substantially shocked material ${ }^{20,21}$. However, strongly shocked lithologies embedded in unshocked lithologies (not as xenoliths) have been rarely observed ${ }^{15,22}$. Previous studies on NWA 7298 (H3.8) and Mokoia (CV3/S1) showed distinctly shocked lithologies within one hand sample ${ }^{22,23}$, which was attributed to a relatively strong impact that produced the different types of rocks simultaneously but spatially separated. A later transport would be required but was not explained in detail. Conceivably, a limited number of discrete impacts may create mixtures of such distinct lithologies, but shock features would be more likely prevalent throughout the lithology if exposed to high pressures during a hypervelocity impact. This manifests itself by the fact that in most meteorites all chondrules show the same degree of deformation. Previous studies do not rule out additional processes such as the active pebble transport observed on asteroid Bennu.

Particle ejection and reaccretion of $\mathrm{mm}^{-}$to $\mathrm{cm}$-sized particles onto the regolith of Bennu ${ }^{1}$, is an important but until recently undiscovered mass transport process on asteroids. According to the OSIRIS-REX observations, the larger events occurred every two weeks, and smaller detected events happened every 12 days. During the time period of observation, no hypervelocity impact events were detected. This implies that hypervelocity impacts are much less frequent than the particle ejection and reaccretion events. Through the latter, about $10^{4}$ to $10^{5}$ particles may be launched per year ${ }^{24}$ with $85 \%$ of them redeposited and the remainder exceeding the escape velocity ${ }^{2}$. Consequently, a large number of pebble-sized fragments would be relocated on Bennu's surface, leading to global and thorough mass transport and regolith mixing. Candidates for the ejection mechanism are low-energy meteoroid impacts, thermal fracturing, and the fallback of ejecta that are consistent with observations ${ }^{24}$. The impact and thermal models are both able to produce centimeter-scale and smaller particles with a speed of a few meters per second ${ }^{25,26}$, which is roughly similar to observed particle velocities $\left(0.05\right.$ to $\left.>3.3 \mathrm{~m} \mathrm{~s}^{-1}\right)$, though the velocities in the thermal model are a little lower $\left(\sim\right.$ maximum of $\left.2 \mathrm{~m} \mathrm{~s}^{-1}\right)$ than the maximum observed. Meanwhile, the size distribution of ejecta derived from the thermal model matches that of the observations well ${ }^{26}$. It is possible that thermal fracturing breaks up boulders into smaller particles that are then ejected by meteoroid bombardment ${ }^{2}$.

Spectral data of Bennu suggests a $\mathrm{CM}$ composition ${ }^{6}$, similar to $\mathrm{AZ}$, and therefore thermal stressing and mass transport may have also occurred on the AZ parent body and mixed the compact AZ lithology with the regular one. We compared the size distribution of compact $A Z$ fragments with that of Bennu ejecta and found a notable match (Fig. 4) that indicates a similar breakup/transport mechanism. Both datasets are truncated at the lower end due to the observational detection limits of OSIRIS-REx and sample processing in the laboratory for AZ, respectively. To better understand such activity on asteroids and its potential to transport mass globally on the AZ parent body, we conducted a Monte Carlo analysis. Tens of thousands of fragments were released from the surface of asteroids with 1-100 Bennu radii. The ejecta redeposited onto the surface after orbiting the asteroid several times or not or escaped the parent body's gravity directly. Their trajectories were recorded, and the efficiency of global transport was evaluated. To quantify the particle relocation, we used the concept of displacement angle as the central angle between the launching site and landing site. A large displacement angle represents a global transport, otherwise a 
local one, and we arbitrarily set a threshold equal to $\Pi / 4$ to distinguish between the two types of transport. Our model yields a pronounced equatorial excess of particle ejections on a Bennu-sized body (Supplementary Fig. 4), consistent with the spacecraft observation ${ }^{1,2}$. The model also predicts that the particle ejection and redeposition process operates as an effective mixing process on asteroids with a radius of up to 30-50 Bennu radii (see Fig. 5). Though the transport became less global with increasing asteroid size, a significant number of ejecta were still moved globally even when the asteroid was 50 times larger than Bennu.

This supports the hypothesis that such a process occurred on the AZ parent body. Conventionally, the deformation that results from impacts is a localized phenomenon, and specimens from the same meteorite fall have the identical petrofabric in most cases. Nonetheless, if the active pebble transport occurred on the AZ parent body, it was able to throw out compact AZ fragments globally from the impact basin. After mass transport, compact AZ fragments were mixed into unshocked regular AZ lithology, and later impacts consolidated the breccia and ejected it to Earth. The consolidating impact may have resulted in the final ejection.

Based on our observations we propose the following scenario for the formation of AZ (Fig. 6). (1) A hypervelocity impact caused the deformation of chondrules and formed cracks in matrix (2-20 $\mu \mathrm{m}$ wide). (2) A pebble transport process such as observed on Bennu ejected compact fragments that reaccreted into unshocked regolith later. (3) The absence of precipitates in cracks implies that no significant aqueous alteration happened after that and that lithification of the AZ breccia happened through one or multiple later impacts.

The high frequency of pebble transport on Bennu and AZ-like asteroids is at odds with the low frequency of occurrence of compact fragments in unshocked lithology seen in carbonaceous chondrites. We argue that this is an observational bias for three reasons: first, the abundance of compact material is relatively low (3.2 wt\% in this study), and most studies do not usually survey sample volumes as large as in this study. Second, if the process is common on active asteroids like Bennu but not others we will observe the resulting lithology in meteorites ejected from this type of asteroid but not in meteorites from inactive parent bodies, which may be more common. Third, traditional techniques such as polished sections only provide information from a small sample of the whole rock. In fact, different shock stages of Murchison are reported $3,16,18$, and the average 3D aspect ratio of chondrules in Murchison ranges from $1.75 \pm 0.39^{14}$ to $1.54 \pm 0.22^{10}$ and $1.30 \pm 0.15$ (this study), whereas the chondrule aspect ratio in $2 \mathrm{D}$ sections is typically less than $1.2^{8}$. All the evidence is consistent with an active parent body for Murchison as well. We predict that other carbonaceous chondrite breccias, in particular $\mathrm{CM}$ chondrites, as well as the mission-returned samples from the asteroid Bennu, may also contain compact fragments embedded in an unshocked lithology. Studying other carbonaceous breccias will provide new insights into the diversity and relative importance of this and other surface processes on active asteroids.

\section{Methods}


Sample preparation. A large $1.894 \mathrm{~kg}$ fragment of Aguas Zarcas was recovered rapidly after its fall before rain, purchased by Terry Boudreaux and donated to the Field Museum of Natural History. At the Field Museum this specimen, FMHN ME 6112, is stored in a stainless-steel cabinet in an inert nitrogen atmosphere at room temperature. $79 \mathrm{~g}$ fragments were separated from a large sample of AZ, FMHN ME 6112 with cleaned stainless-steel tools in a nitrogen-filled glove bag. We used freeze-thaw disintegration as the first step of an effort to separate objects of interest including refractory minerals, isolated olivine grains, and presolar grains from the fine-grained matrix and organic matter of $A Z$. The selected pieces were roughly divided into ten $\sim 8$-gram chunks in ultrapure water $(18.2 \mathrm{M} \Omega \bullet \mathrm{cm}$ electrical resistivity; MilliQ) and each was disintegrated using alternating cycles of liquid nitrogen and $50^{\circ} \mathrm{C}$ water. Typically, 30 cycles can break down the matrix of a CM2 chondrite like Murchison into powder. With AZ, most fragments were disaggregated within 50 cycles, however, more than 10 sub-cm-sized fragments (3.2 wt\%) remained intact and showed no signs of mechanical breakdown after 112 cycles. We call these intact fragments "compact AZ".

11 compact AZ fragments and 3 randomly chosen AZ (FMNH ME6110.1) fragments that were not processed by freeze-thaw were $\mu \mathrm{CT}$-scanned at the PaleoCT facility of the University of Chicago. The fragments of compact $A Z$ are named from CF- 1 to $C F-11$, and the randomly selected $A Z$ fragments are named from RF-1 to RF-3.

X-ray microtomography. We scanned all AZ samples (CF-1 to CF-11 and RF-1 to RF-3) at the University of Chicago's PaleoCT Lab, on a GE v|tome|x S model micro-CT scanner using the $240 \mathrm{kV}$ microfocus tube. The fragments were mounted in a $15 \mathrm{ml}$ tube and scanned at a spatial resolution (or voxel size) of $17.028 \mu \mathrm{m}$. An $80 \mathrm{kV}$ voltage and $220 \mu \mathrm{A}$ current were used with an image acquisition time of $500 \mathrm{~ms}$ per frame. Three frames were captured and averaged for each position to reduce noise and a $0.2 \mathrm{~mm}$ Cu filter was used to reduce beam hardening. The total scan time for the tube of specimens was one hour and fifty minutes.

Two of the larger pieces of AZ (one compact AZ, CF-10, $0.591 \mathrm{~g}$; one regular AZ, RF-1, $0.730 \mathrm{~g}$ ) as well as a specimen of Murchison (FMNH ME2644; $1.171 \mathrm{~g}$ ) and of Leoville (FMNH ME2628.2; $1.706 \mathrm{~g}$ ) for comparison, were scanned at the University of Texas High-Resolution X-ray Computed Tomography Facility (UTCT) at higher resolution. These four samples were scanned on a Zeiss Versa 620 at $80 \mathrm{kV}$ and $125 \mu \mathrm{A}$ with varying acquisition time per frame $(40-70 \mathrm{~ms})$ with one frame per position for all samples except Leoville, which had 2 frames per position. The LE3 filter was used for all scans and a beam hardening correction was applied during reconstruction with the scanner software, and the final voxel size of each sample scan was $6.77 \mu \mathrm{m}$ (both AZ fragments; scan time 59 minutes each), $8.47 \mu \mathrm{m}$ (Murchison; scan time 53 minutes) and $11.01 \mu \mathrm{m}$ (Leoville; scan time 74 minutes).

Scanning Electron Microscopy. After $\mu \mathrm{CT}$ scanning the compact fragment CF-10 was embedded in Buehler EpoxiCure 2 epoxy and cross-sectioned parallel to the long axis of the flattened chondrules with a Buehler IsoMet low-speed diamond wafering saw. The section was coarsely polished with diamond Allied High Tech Products Inc. lapping film followed by a final polish with Allied $1 \mu \mathrm{m}$ diamond slurry. The 
polished mount was imaged and mapped with a field-emission TESCAN LYRA3 SEM/FIB equipped with two Oxford Instruments XMax SDD $80 \mathrm{~mm}^{2}$ energy dispersive X-ray spectroscopy (EDS) detectors at the University of Chicago. An EDS map, a backscattered electron (BSE) map, and a secondary electron (SE) map were acquired with an acceleration voltage of $15 \mathrm{kV}$ and a beam current of $470 \mathrm{pA}$ and a typical pixel dwell time of $25 \mu$ s at a nominal spatial resolution of 0.369 and $0.185 \mu \mathrm{m}$ per pixel, respectively. EDS spectra were used to determine the mineral chemistry qualitatively at an accuracy of $<5 \mathrm{wt} \%$. Crosssectional morphology and texture including fractures were examined with the EDS, SE, and BSE maps using Oxford Instruments AZTec software.

Chondrule segmentation and deformation analysis. First, we determined the $\mu \mathrm{CT}$ components in compact AZ by calibrating the CT data by comparing BSE and EDS maps of the polished cross section of compact AZ fragment CF-10 with a matching $\mu \mathrm{CT}$ slice. There are three types of objects in AZ that we identified based on their grayscale values within the $\mu$ CT data (Supplementary Fig. 5): small bright objects without well-defined shapes, light-toned objects, and dark-toned objects. Here we mainly discuss chondrules and neglect irregular clasts. Earlier $\mu \mathrm{CT}$ studies ${ }^{10,14}$ of Murchison show that the brightest components are metal and sulfides such as pentlandite and that light-toned and dark-toned objects are mostly Fe-bearing chondrules/CAls and Fe-poor/Mg-rich chondrules, respectively. SEM data of the polished AZ CF-10 confirm the same $\mu \mathrm{CT}$ components as in Murchison. In the $\mu \mathrm{CT}$ data of Leoville, we only observed dark chondrules and bright metal/sulfides. $\mu \mathrm{CT}$ data of regular AZ shows the same three object types as compact AZ. According to the previous research ${ }^{10,14}$, dark-toned objects (i.e., Mg-rich chondrules) are typically more deformed and display a stronger fabric compared to bright (metal and sulfide) and lighttoned (Fe-bearing chondrules and CAls) objects. Regardless of the reason for this observation, we only delineated and segmented the dark-toned objects in $\mu \mathrm{CT}$ data and calculated the fabric strength to avoid any potential observational bias with objects of different X-ray contrast.

Secondly, we outlined components of interest (here dark-toned chondrules) from the tomographic data set into distinct volumes of interest. We used manual segmentation in 3D Slicer software (http://slicer.org) where we used the "draw" tool to mark chondrules in individual 2D slices, then filled between slices to obtain a 3D visualization ${ }^{27}$. This method is labor-intensive and time-consuming if performed for every chondrule in the dataset. Therefore, we only applied it to small fragments and used a more efficient alternative, the partial segmentation method, to large ones. For partial segmentation, one or more representative cross-sections in each chondrule's orthogonal plane are chosen for segmentation excluding the ambiguous chondrules such as those that are in contact with each other. The effectiveness of this method to accurately calculate the orientation and degree of anisotropy of objects in rocks relative to the full segmentation has been examined and confirmed ${ }^{10}$. In this study, we used the whole segmentation method for samples scanned at University of Chicago, as these data sets are small due to their lower resolution, as well as for Leoville, where our scanned volume contains only a few chondrules due to their relatively large size. For the remaining datasets, we used the partial segmentation method. 
Thirdly, after segmentation with 3D Slicer, we exported all the segments to DICOM files, loaded them into Fiji, and converted them to TIFF files. For each chondrule we used Blob3D 28,29

(http://www.ctlab.geo.utexas.edu/software/blob3d/) to determine the size, location, and orientation information via best-fit ellipsoids to either the full segmentation or partial segmentation via a set of orthogonal planes. Orientation biasing can occur when an object covers only a few voxels. To avoid that, we removed objects with a short axis of less than 3 voxels ${ }^{10}$. In order to make the data volume manageable, we divided each large tomographic data set into several subvolumes and segmented chondrules within each individual subvolume. This enabled faster processing of the data and a reduction in file size. We segmented 825 dark-toned objects in total. Parameters of best-fit ellipsoids to each object are shown in Supplementary Table 1.

Fabric analysis of the tomographic data in this work follows an established method ${ }^{10}$, and further details regarding parameter calculations reported in Supplementary Table 1 can be found in that work. Here we briefly introduce the quantitative analysis of deformation. We take the direction vectors of a set of axes of the fitted ellipsoids as an example. These directions are plotted on stereonets in a lower hemisphere projection, and the forming pattern is used to test whether the orientations are non-random. Meanwhile, Woodcock and Naylor ${ }^{13}$ defined $K$ and $C$ parameters to describe the shape and strength respectively of a fabric. An orientation tensor ( $3 \times 3$ matrix) is calculated from the above direction vectors, and three eigenvectors of the tensor are defined as $S_{1}, S_{2}, S_{3}$. $K$ is defined as $K=\ln \left(S_{1} / S_{2}\right) /\left(S_{2} / S_{3}\right)$, and $C$ is defined as $C=\ln \left(S_{1} / S_{3}\right)$. $K$ ranges from zero (girdle or 'great circle' distribution on a stereonet for $K<1$ ) to infinite (cluster distribution for $K>1)^{30}$. $C$ ranges from zero (no fabric) to 4 or above (strong fabric) and is manifested as the concentration of data points on a stereonet ${ }^{30}$. Supplementary Fig. 6 illustrates $K$ and $C$ parameters and the chondrule orientations for 4 types of rocks in this study.

Shock Pressure determination. Previous studies 8,18 used Murchison and Allende in shock-recovery experiments to build up empirical relationships between aspect ratio and shock pressure in a single impact shock event. In Murchison, $10 \mathrm{GPa}$ was a threshold over which the aspect ratio started transferring from $\sim 1.2$ to 1.5 (see Fig. 3). Meanwhile, $20 \mathrm{GPa}$ was another threshold over which the aspect ratio kept approximately constant. Specifically, the aspect ratios of chondrules in an impacted sample had a large range, but the distribution of the aspect ratios moved clearly with an increasing shock pressure. Accordingly, the mean values of those ratios rose. Also, the aspect ratios of unshocked Murchison's and Allende's chondrules were not counted for linear regression. In the shock-recovery experiments, the recovered samples were cut along the shock compacting axis, such that the mean $2 \mathrm{D}$ aspect ratio of chondrules in the section was most comparable to the mean ratio of the longest axis length to the shortest axis length in our 3D model (called 3D aspect ratio).

Lithostatic pressure Model. At depth within a spherical asteroid, the force balance is as follows: $G M / r^{2} \cdot \rho 4 \Pi r^{2} d r=-4 \Pi r^{2} d P$, when $r$ is the radial distance from the center of the parent body, $G$ is the gravitational constant, $M$ is the mass of the material below $r, \rho$ is the density, $P$ is the pressure. The left part is the gravitational force of a shell with a width of $d r$ at a radial distance $r$ from the center, and 
the right part is the supporting force provided by the pressure gradient. Due to $M=4 / 3 \Pi r^{3} \rho$, the simplified equation of force balance is $d P=-4 / 3 \Pi G \rho^{2} r d r$. The solution is $P=2 / 3 \Pi G \rho^{2}\left(R^{2}-r^{2}\right)$, where $R$ is the radius of the parent body. When $r=0, P$ reaches the maximum that is the pressure at the center. Because most stony meteorites have densities ${ }^{31,32}$ on the order of 3 to $4 \mathrm{~g} \mathrm{~cm}^{-3}$, we take $\rho=3.5 \mathrm{~g}$ $\mathrm{cm}^{-3}$ in the model. We consider two cases to visualize the pressure profiles in meteoritic parent bodies. One is the maximum pressure (center pressure) for spherical objects with different sizes; the other is the depth-pressure profile for a 100-km-sized body (Supplementary Fig. 3). The calculated maximum pressure for the Moon is $5.2 \mathrm{GPa}$, and most petrological experiments and seismic detections all support a $\sim 5 \mathrm{GPa}$ pressure at the lunar core or core-mantle boundary ${ }^{33-35}$. The maximum pressure for a $100-\mathrm{km}-$ sized body is $<0.02 \mathrm{GPa}$.

Monte Carlo Model. The movement of ejecta on Bennu is controlled by multiple forces such as Bennu's gravity, solar radiation, reflected pressure, Poynting-Robertson effect, etc. The gravitational force in the vicinity of Bennu is 2 to 6 orders of magnitude higher than the other forces ${ }^{2}$, therefore, and for simplicity, we consider it as the only driving force in our model. Besides this, we set up initial conditions that include initial velocities of particles, launch position, and rotation of the central body. The observed velocity of Bennu ejecta ranges from $0.05 \mathrm{~m} \mathrm{~s}^{-1}$ to $>3.3 \mathrm{~m} \mathrm{~s}^{-1}$, and the observations may not include all particles especially fast-moving ones. Thus, we take $0.05-5 \mathrm{~m} \mathrm{~s}^{-1}$ as the initial velocity range. The particles can be ejected from anywhere on the surface but were more frequently observed from low latitudes. We adopt the distribution of ejection sites from Chesley et al. ${ }^{2}$ to our model. Generally, the spin period of asteroids decreases with their size and clusters between 2-12 hours ${ }^{36}$. The rotation period for Bennu is 4.3 hours, and we apply this to all the simulations in this study.

First, we modeled particle movement on a spherical body whose mass, bulk density, and radius are the same as that of Bennu. 50,000 particles were released, and only those with low velocities $\left(<0.35 \mathrm{~m} \mathrm{~s}^{-1}\right)$ fell back on the surface. Supplementary Fig. 4 depicts the distribution of the sine of latitude for ejecta deposition. We also ran our model with larger asteroids with 10 to 100 Bennu radii. Bennu's bulk density is low $\sim 1.26 \mathrm{~g} \mathrm{~cm}^{-3}$ because it is a rubble pile asteroid with a high porosity ${ }^{37}$. Nevertheless, the fragment density should be close to that of its meteorite analog AZ $\left(\sim 2.4 \mathrm{~g} \mathrm{~cm}^{-3}\right)$. Here we argue that $2.4 \mathrm{~g} \mathrm{~cm}^{-3}$ is the approximate upper limit for such carbonaceous chondrites because it ignores the pore space in the parent body. The mean densities of $\mathrm{C}, \mathrm{S}$, and $\mathrm{M}$ class asteroids are $1.38,2.71$, and $5.32 \mathrm{~g} \mathrm{~cm}^{-3}$, respectively, from calculations ${ }^{38}$. We adopted 1.26 and $2.4 \mathrm{~g} \mathrm{~cm}^{-3}$ separately in our model.

\section{References}

1. Lauretta, D. S. et al. Episodes of particle ejection from the surface of the active asteroid (101955) Bennu. Science 366, \#eaay544 (10 pp) (2019).

2. Chesley, S. R. et al. Trajectory estimation for particles observed in the vicinity of (101955) Bennu. J. Geophys. Res. Planets. 125, \#e2019JE006363 (33 pp) (2020), 
3. Rubin, A. E. Collisional facilitation of aqueous alteration of CM and CV carbonaceous chondrites. Geochim. Cosmochim. Acta 90, 181-194, (2012).

4. Bischoff, A., Scott, E. R. D., Metzler, K. \& Goodrich, C. A. Nature and origins of meteoritic breccias. Meteorites and the Early Solar System II, 679-712 (2006).

5. Bischoff, A., Schleiting, M., Wieler, R. \& Patzek, M. Brecciation among 2280 ordinary chondrites constraints on the evolution of their parent bodies. Geochim. Cosmochim. Acta 238, 516-541, (2018).

6. Hamilton, V. E. et al. Evidence for widespread hydrated minerals on asteroid (101955) Bennu. Nat. Astron. 3, 332-340 (2019).

7. Kerraouch, I. et al. The polymict carbonaceous breccia Aguas Zarcas: A potential analog to samples being returned by the OSIRIS-REx and Hayabusa 2 missions. Meteorit. Planet. Sci. 56, 277-310 (2021).

8. Tomeoka, K., Yamahana, Y. \& Sekine, T. Experimental shock metamorphism of the Murchison CM carbonaceous chondrite. Geochim. Cosmochim. Acta 63, 3683-3703, (1999).

9. Cain, P. M., McSween H. Y. Jr. \& Woodward, N. B. Structural deformation of the Leoville chondrite. Earth Planet. Sci. Lett. 77, 165-175 (1986).

10. Hanna, R. D., Ketcham, R. A., Zolensky, M. \& Behr, W. M. Impact-induced brittle deformation, porosity loss, and aqueous alteration in the Murchison CM chondrite. Geochim. Cosmochim. Acta 171, 256282, (2015).

11. Sneed, E. D. \& Folk, R. L. Pebbles in the lower Colorado river, Texas a study in particle morphogenesis. J. Geol. 66, 114-150 (1958).

12. Graham, D. J. \& Midgley, N. G. Graphical representation of particle shape using triangular diagrams: An excel spreadsheet method. Earth Surf. Proc. Land. 25, 1473-1477 (2000).

13. Woodcock, N. \& Naylor, M. A. Randomness testing in three-dimensional orientation data. J. Struct. Geol. 5, 539-548 (1983).

14. Lindgren, P., Hanna, R. D., Dobson, K. J., Tomkinson, T. \& Lee, M. R. The paradox between low shockstage and evidence for compaction in $\mathrm{CM}$ carbonaceous chondrites explained by multiple lowintensity impacts. Geochim. Cosmochim. Acta 148, 159-178, (2015).

15. Friedrich, J. M. et al. Relationships among physical properties as indicators of high temperature deformation or post-shock thermal annealing in ordinary chondrites. Geochim. Cosmochim. Acta 203, 157-174 (2017).

16. Scott, E. R. D., Keil, K. \& Stöffler, D. Shock metamorphism of carbonaceous chondrites. Geochim. Cosmochim. Acta 56, 4281-4293, (1992).

17. Rubin, A. E. \& Swindle, T. D. Flattened chondrules in the LAP 04581 LL5 chondrite: Evidence for an oblique impact into LL3 material and subsequent collisional heating. Meteorit. Planet. Sci. 46, 587600, (2011). 
18. Nakamura, T., Tomeoka, K., Takaoka, N., Sekine, T. \& Takeda, H. Impact-induced textural changes of CV carbonaceous chondrites: Experimental reproduction. Icarus 146, 289-300, (2000).

19. Stöffler, D., Keil, K. \& Scott, E. R. D.. Shock metamorphism of ordinary chondrites. Geochim. Cosmochim. Acta 55, 3845-3867 (1991).

20. Kojima, T., Yatagai, T. \& Tomeoka, K. A dark inclusion in the Manych LL (3.1) ordinary chondrite: A product of strong shock metamorphism. Antarctic Meteor. Res. 13, 39 (2000).

21. Kring, D. A. et al. Portales Valley: A meteoritic sample of the brecciated and metal-veined floor of an impact crater on an H-chondrite asteroid. Meteorit. Planet. Sci. 34, 663-669, (1999).

22. Friedrich, J. M., Weisberg, M. K. \& Rivers, M. L. Multiple impact events recorded in the NWA $7298 \mathrm{H}$ chondrite breccia and the dynamical evolution of an ordinary chondrite asteroid. Earth Planet. Sci. Lett. 394, 13-19 (2014).

23. Ohnishi, I. \& Tomeoka, K. Dark inclusions in the Mokoia CV3 chondrite: Evidence for aqueous alteration and subsequent thermal and shock metamorphism. Meteorit. Planet. Sci. 37, 1843-1856, (2002).

24. Hergenrother, C. W. et al. Photometry of particles ejected from active asteroid (101955) Bennu. J. Geophys. Res. Planets 125, \#e2020JE006381 (15 pp) (2020).

25. Bottke, W. F. et al. Meteoroid impacts as a source of Bennu's particle ejection events. J. Geophys. Res. Planets 125, \#e2019JE006282 (16 pp) (2020).

26. Molaro, J. L. et al. Thermal fatigue as a driving mechanism for activity on asteroid Bennu. J Geophys. Res. Planets 125, \#e2019JE006325, (24 pp) (2020).

27. Fedorov, A. et al. 3D Slicer as an image computing platform for the Quantitative Imaging Network. Magn. Reson. Imaging 30, 1323-1341 (2012).

28. Ketcham, R. A. Three-dimensional grain fabric measurements using high-resolution X-ray computed tomography. J. Struct. Geol. 27, 1217-1228 (2005).

29. Ketcham, R. A. Computational methods for quantitative analysis of three-dimensional features in geological specimens. Geosphere 1, 32-41 (2005).

30. Turner, F. J. \& Weiss, L. E. Structural analysis of metamorphic tectonites. (McGraw-Hill. New York. US, 1963).

31. Britt, D. T. \& Consolmagno, G. Stony meteorite porosities and densities: A review of the data through 2001. Meteorit. Planet. Sci. 38, 1161-1180 (2003).

32. Consolmagno, G. J., Britt, D. T. \& Macke, R. J. The significance of meteorite density and porosity. Geochem. 68, 1-29 (2008).

33. Rai, N. \& van Westrenen, W. Lunar core formation: New constraints from metal-silicate partitioning of siderophile elements. Earth Planet. Sci. Lett. 388, 343-352, (2014).

34. van Kan Parker, M. et al. Neutral buoyancy of titanium-rich melts in the deep lunar interior. Nat. Geosci. 5, 186-189 (2012). 
35. Weber, R. C., Lin, P.-Y., Garnero, E. J., Williams, Q. \& Lognonné, P. Seismic detection of the lunar core. Science 331, 309-312 (2011).

36. Walsh, K. J. Rubble pile asteroids. Annu. Rev. Astron. Astr. 56, 593-624 (2018).

37. Lauretta, D. S. et al. The OSIRIS-REx target asteroid (101955) Bennu: Constraints on its physical, geological, and dynamical nature from astronomical observations. Meteorit. Planet. Sci. 50, 834849, (2015).

38. Krasinsky, G. Hidden mass in the asteroid belt. Icarus 158, 98-105, (2002).

\section{Declarations}

\section{Funding:}

NASA's Emerging Worlds program 80NSSC21K0389 to PRH

NASA's Emerging Worlds program 80NSSC21K0374 to AMD

NSF grant EAR-1762458 to UTCT Facility

\section{Author contributions:}

X.Y. and P.R.H. conceived the study and wrote the paper with input from all authors.

R.D.H provided expertise on the data processing, interpretation, and visualization. A.M.D contributed to the investigation and Monte Carlo model. A.I.N. conducted the first $\mu \mathrm{CT}$ scanning of samples. X.Y. and P.R.H prepared the samples for SEM/EDS analysis, and A.M.D helped explain the data.

Competing interests: All other authors declare they have no competing interests.

Data and materials availability: All data needed to evaluate the conclusions in the paper are present in the paper and/or the Supplementary Information. The original $\mu C T$ data and Monte Carlo simulations with $M A T L A B$ are available upon request from $X Y$.

\section{Figures}




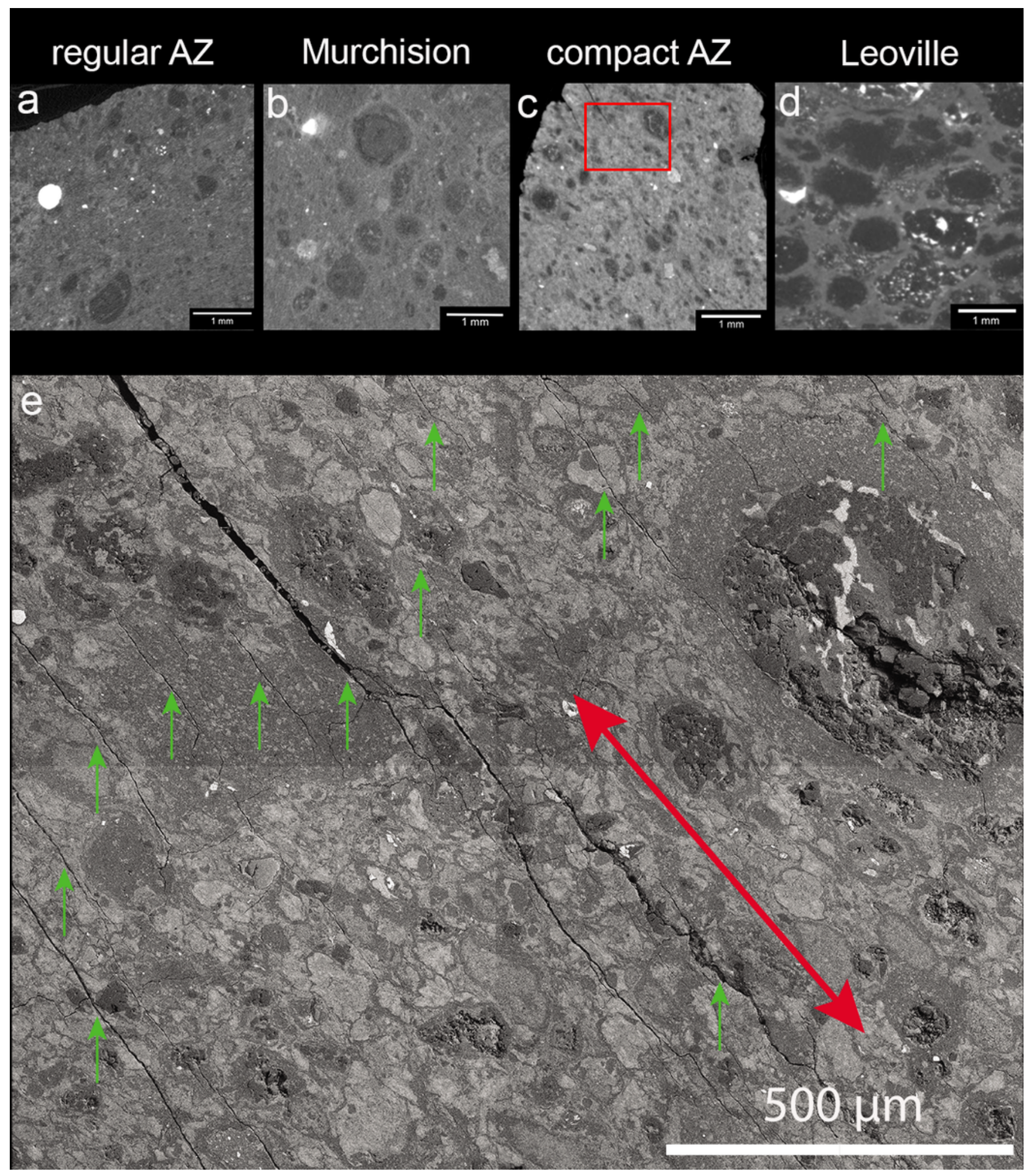

\section{Figure 1}

Slices from $\mu C T$ image stacks of 4 types of rocks and BSE image of a polished compact AZ fragment. ad, $\mu C T$ images of four samples regular AZ, Murchison, compact AZ, and Leoville. Pronounced elongation and orientation of chondrules are observed in $\mathbf{c}$ and $\mathbf{d}$. e, BSE image of the approximately same area as the red rectangle in $\mathbf{c}$. Foliation plane and chondrule flattening direction are indicated by the red arrow 
and fractures are indicated by the green arrows. In e bright sulfide veins in the chondrule in the upper right are unparallel to the direction of chondrule flattening.

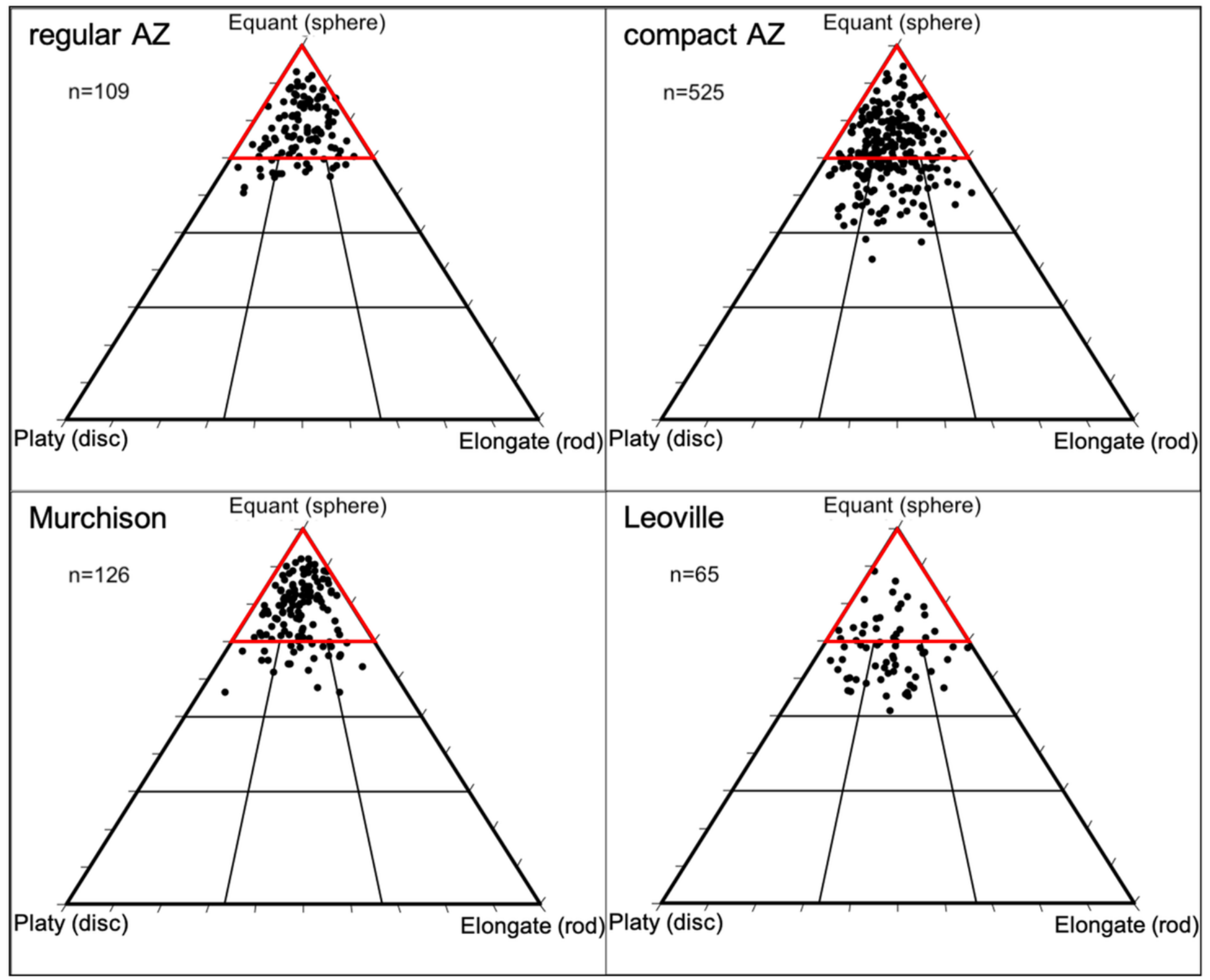

Figure 2

Chondrules fitted with ellipsoid shapes. The ellipsoid axis lengths are used to plot each chondrule shape within the triangle whose apices represent the idealized particle shapes. The fraction of data points in the top sub-triangle (red) is $82.6 \%$ for regular AZ, $81.0 \%$ for Murchison, $56.0 \%$ for compact $A Z$, and $35.4 \%$ for Leoville. $\mathrm{n}$ is the number of best-fitted ellipsoids. 

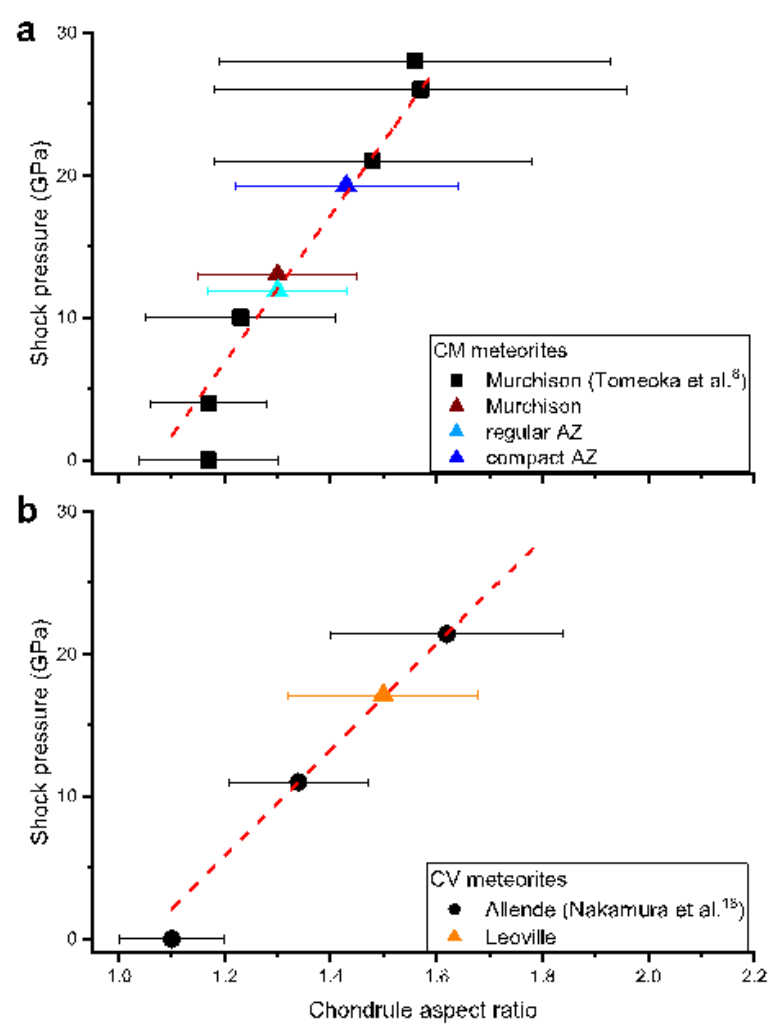

Figure 3

Plot of the relationship between aspect ratio and shock pressure for $\mathrm{CM}$ meteorites in a and CV meteorites in b. Fitted ellipsoids from regular AZ and Murchison have the same mean aspect ratio; we moved the Murchison data point to the upper to distinguish it from the regular AZ data point. The Allende and Murchison data from Tomeoka et al. ${ }^{8}$ and Nakamura et al. ${ }^{18}$ were used to create standard curves (red dashed lines), and the last two Murchison date points in a with high shock pressures were not counted because of the approximately constant aspect ratio. Triangles are used for our data. Error bars denote the data range as one standard deviation not data uncertainties, so the mean value is still useful in determining shock pressures. 


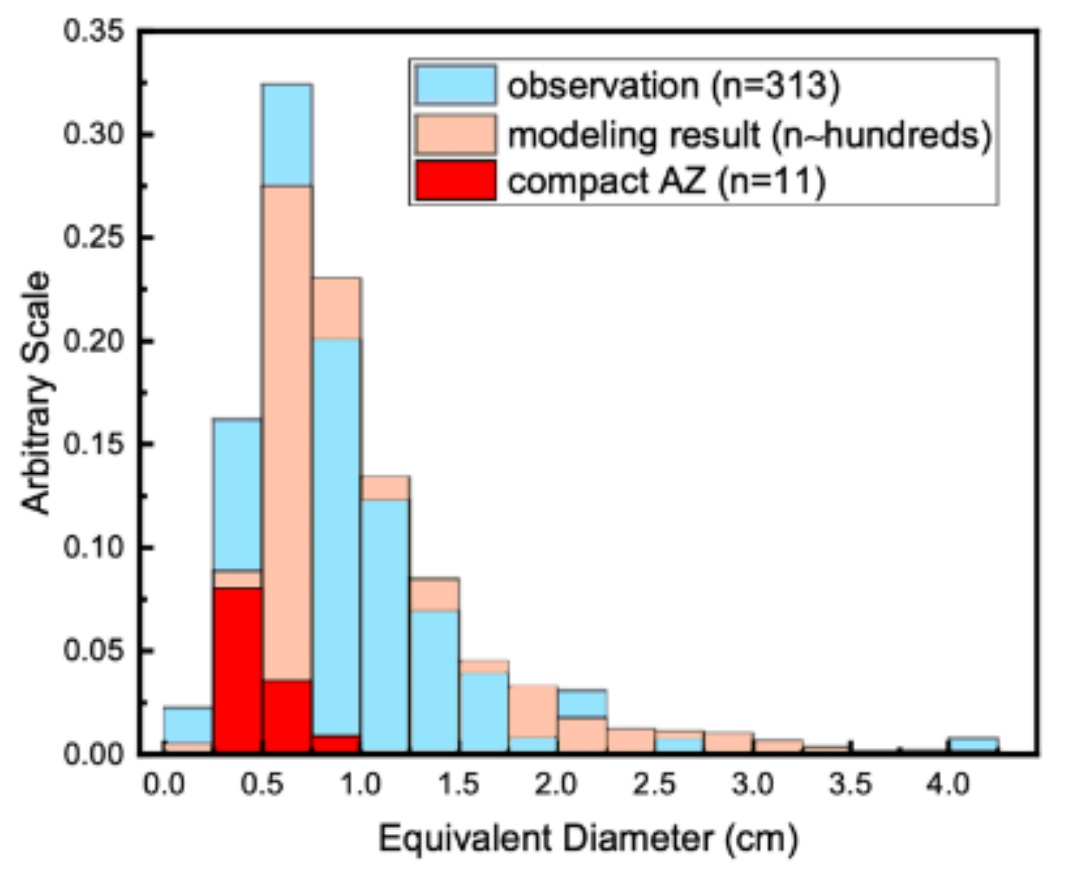

Figure 4

Distribution of volume-equivalent spherical diameters for particles ejected from asteroid Bennu and for the compact $\mathbf{A Z}$ fragments. The blue represents the observation based on the combined information from absolute magnitude and area-to-mass ratio ${ }^{2}$. The beige represents the size distribution of thermally fractured fragments according to the exfoliation model ${ }^{26}$. The red bars depict the size distribution of compact AZ fragments with an arbitrary scale. The compact AZ samples correspond to the typical size of Bennu ejecta, though we only collected 11 fragments. 

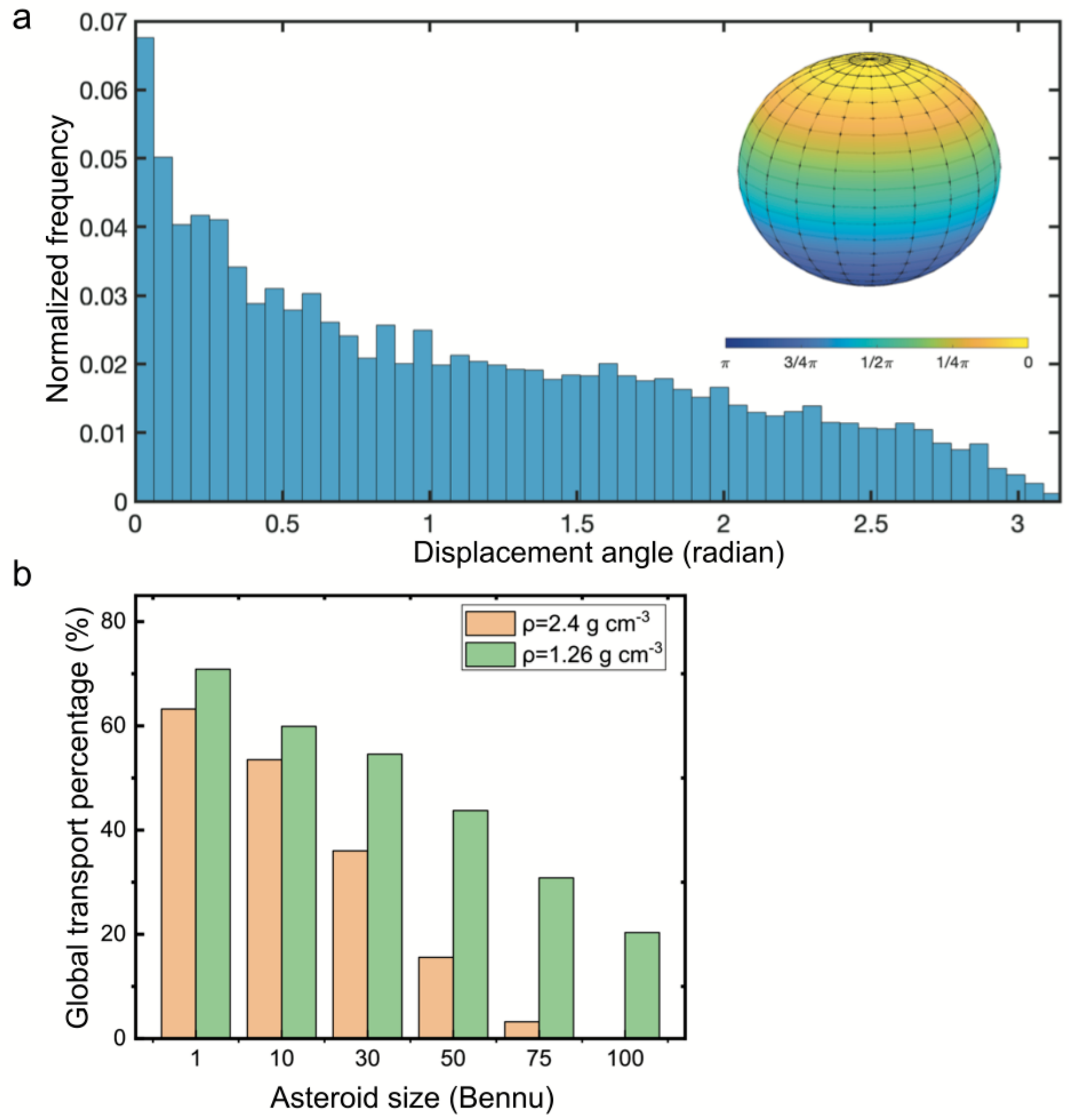

Figure 5

Characteristics of modeled ejecta. a, Distributions of displacement angle for asteroids with a radius of 30 Bennu radii and a bulk density of $1.26 \mathrm{~g} \mathrm{~cm}^{-3}$. The initial velocity is between 0.05 to $5 \mathrm{~m} \mathrm{~s}^{-1}$, and the launching site ranges from equator to arctic. The displacement angle is the central angle between the launching site and landing site and illustrated by the color sphere. If a particle is ejected from the north pole and lands elsewhere, the color at the landing site denotes the displacement angle. $\mathbf{b}$, The percentage 
of ejecta that were transported globally not locally (displacement angle $>\pi / 4$ ). The model considers the bulk density of asteroid Bennu $\left(1.26 \mathrm{~g} \mathrm{~cm}^{-3}\right)^{37}$ and Aguas Zarcas bulk density $\left(2.4 \mathrm{~g} \mathrm{~cm}^{-3}\right)$ separately.

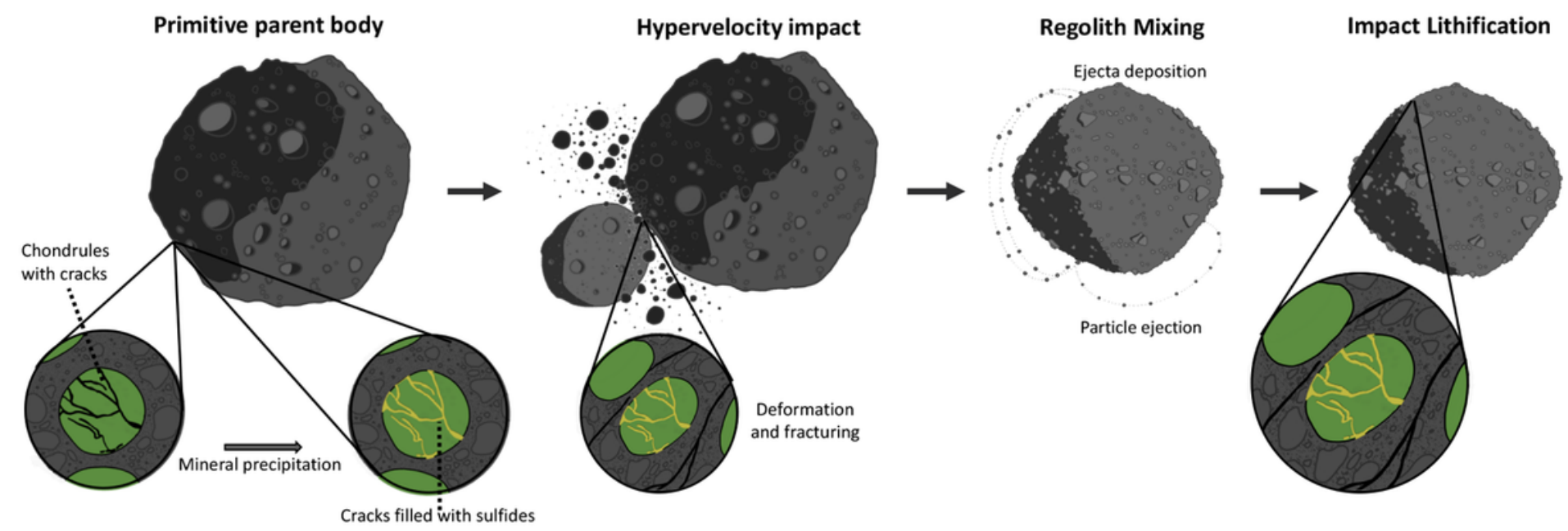

Figure 6

Schematic portrayal of the history of the formation of the Aguas Zarcas chondrite. Fractures were generated in chondrules before or during the accretion of the parent body and were filled simultaneously by shock mobilization or later by thermal/aqueous alteration. Then a hypervelocity impact caused chondrule flattening and fracturing in the matrix, and the compact AZ lithology was formed. Particle ejection and reaccretion events redistributed rock fragments with distinct lithologies, mixing compact AZ into regular AZ. Later impacts consolidated the mixed lithologies and resulted in the final ejection.

\section{Supplementary Files}

This is a list of supplementary files associated with this preprint. Click to download.

- YangetalNASupplementary.docx 